\title{
Design and Analysis of an Interactive MOOC Teaching System Based on Virtual Reality
}

\author{
https://doi.org/10.3991/ijet.v13i07.8790 \\ Yongfei Zhang $\left({ }^{\square}\right)$ \\ Dali University, Dali, Yunnan, China \\ yongfeizhan $3847 @ 126 . \mathrm{com}$ \\ Jianshu Chen, Dan Miao, Chen Zhang \\ Honghe Health Vocational College, Mengzi, Yunnan, China
}

\begin{abstract}
In order to solve the problem of lack of immersion in the MOOC curriculum, through research on key technologies, a MOOC teaching interactive system based on virtual reality was constructed to improve it. Through the decomposition of complex objects or the addition of blank sub-objects, the system built corresponding collision detectors to achieve more realistic collision detection effects. The immersive and interactive nature of the virtual user in the virtual scene was enhanced. The results showed that the system used the immersive, interactive and constructive sense of virtual reality to build people's interest in learning. Therefore, the MOOC teaching interactive system based on virtual reality has a good sense of immersion, interaction and conceivability.
\end{abstract}

Keywords-virtual reality; MOOC teaching; interactive system; 3D modeling

\section{$1 \quad$ Introduction}

With the development of communication technology and the increasing popularity of communication infrastructure, distance learning based on network multimedia has become the best way to satisfy people's lifelong learning needs. To cater to people's ever-increasing demand for learning content, such as diversification and globalization, and the time awareness, the massive online open course (MOOC) learning mode was developed with the micro-course as a unit has emerged. MOOC provides the same platform for lifelong learning for people with different regions, cultures, and economic levels. It opened the door for more people to experience the higher learning that had been left behind by many people. Anyone can register and log on to the MOOC education platform via the Internet and choose any MOOC course that they are interested in. After learning, they obtain the certificate of the course through corresponding examination. MOOCs are rapidly sought after by people for their free, open and unrestricted features. The number of students participating in MOOCs worldwide and the number of universities are all increasing. With the rapid development of MOOCs, their shortcomings have become increasingly prominent. Although it satisfies the people's desire for knowledge, the boring network teaching model makes the learning 
process lack immersion, interactivity and interest. However, due to its strong sense of immersion, interaction and interest, virtual reality technology can make up for the lack of mainstream MOOC teaching mode. Therefore, research on the MOOC teaching model based on virtual reality has a strong practical significance. The MOOC communication platform based on virtual reality is a bold attempt to combine modern information science technology with a new type of educational philosophy. It inherits the characteristics of the large-scale, free and open education model of MOOC. It combines virtual reality technology to make up for the lack of immersion, interactivity and real-time performance in existing online education. This article aims to study how to combine virtual reality technology with MOOC to supplement the existing MOOC teaching model and provide learners with a good autonomous learning platform.

\section{Literature review}

The term MOOC was coined in 2008 by Dave Cormier from the University of Prince Edward Island (Canada) and a senior researcher Bryan Alexander worked in the Canadian Institute of Humanities Educational Technology Application. MOOC achieved unprecedented development in the United States in 2012. In foreign countries, the design of MOOC courses is mainly divided into two types: First, centered on institutions with higher learning, popular courses in universities are published on the MOOC learning platform. Second, users can independently publish course on the MOOC learning platform to achieve the dissemination of educational resources. Udocity, Coursera and edx are the major MOOC learning platforms established around universities. The user can publish the representative model of the course such as Udemy and P2PU. Borras-Gene O proposed the concept of gamification and MOOC integration in 2016 and applied it to the curriculum design. The research results showed that the combination of MOOC and gamification can improve the learning interest of course learners [1]. In the same year, Hone K. S conducted relevant investigation and analysis on the influencing factors of CMOOC design and implementation [2]. In China, in 2014, Zhu. Y. H. pointed out that the nature and trajectory of online education should be understood from the perspective of education transformation in the era of digital knowledge economy. After the short-term interference, it will eventually be positioned in online courses and online education systems [3]. Lingling $\mathrm{S}$ and other scholars of Tsinghua University conducted a systematic analysis of the characteristics and trends of online education from 2008 to 2014. They also pointed out that MOOCs will gradually be integrated into online education teaching and learning, and provide an open management Mode [4]. Jia H studied the problems encountered in the development of MOOCs in China in 2014 [5]. In 2018, Li C proposed a new hybrid network model. This model was applied to online learning based on the MOOC platform for more than 9,400,000 students' sustainable development related courses [6].

The application of virtual reality technology [7] in the field of education not only solves the shortage of experimental resources in the educational field [8] and the 
shortage of experimental funds, but also provides students with opportunities for exploratory experiments. Because the experimental equipment and the experimental environment are virtual simulations, the experimenter does not need to consider the damage factors of the experimental equipment and the occupation factors of the experimental scene but can freely attempt to carry out exploratory experiments as user likes. This kind of experimental pattern not only deepens the experimenter's understanding of the learned knowledge, but also greatly enhances the experimenter's ability to independently innovate and find solutions to problems.

In this paper, the existing MOOC teaching mode and virtual reality technology are studied, aiming to explore how to apply virtual reality technology on the MOOC teaching mode. Combined the advantages of the two, it analyzes and evaluates existing problems in the MOOC teaching model, such as high student drop-out rate, zero interaction between teachers and students, single learning method and long feedback period. Finally, the corresponding solutions are proposed.

\section{Methodology}

\subsection{Overview of MOOC teaching mode}

The MOOC teaching model is the development and reform of the traditional education model with the development of the Internet. Its essence is to realize large-scale learning and knowledge sharing through the Internet. MOOC maximizes the collection of high-quality online courses resources around the world, so that learners can achieve online education through the computer network. The success of the MOOC teaching model has triggered a revolution in the field of education, breaking the traditional teaching model. Taking tiny knowledge points as a unit, the problem of the learner's scattered and random learning time is better solved. The MOOC learning platform is not only a gathering of learning resources and learners, but also a way to use the same knowledge to connect teachers with learners. The MOOC teaching mode is an online course teaching mode that is openly acquired through the Web and has large-scale interactive participation. Compared with the traditional teaching mode, it has a large-scale, open, and networked nature.

\subsection{Research of MOOC teaching mode}

The MOOC teaching model is learner-centered. Learners can customize the learning goals according to their own situation, organize the learning structure and choose the learning course. Compared with traditional forms of teaching, it pays more attention to learners' initiative and autonomy. Different from the traditional teacher-led approach, education model that the student passively accepts the knowledge has more advantages. MOOC allows people who have learning desires to use the highest quality educational resources to learn at low cost and free of charge. It is changing the traditional way of education for thousands of years, enabling lecturers and learners to interact across time and space, and fundamentally changing the way of knowledge 
acquirement. The current development of MOOC has evolved into two types: cMOOC based on connectivism learning theory and xMOOC teaching model based on behavioral learning theory. The cMOOC teaching model is created on the basis of connectivism learning theory. It emphasizes that knowledge is connected in the way of network, that is, information exists in each node of the learning network. Learners can establish network connections between knowledge nodes. Finally, a learning network is achieved to help learners build knowledge and enhance their abilities.

As shown in figure 1, starting from the interests of learners, it is more conducive to supporting the construction of collaborative dialogues and collaborative knowledge. The cMOOC teaching model is shown in figure 1. It is shown that the cMOOC teaching model is more inclined to teachers-oriented. For example, the teachers propose the topics, provide resources, and organize activities. They guide learners to participate in discussions and exchanges so as to achieve the effect of building a knowledge network. The xMOOC teaching model is developed on the basis of the cMOOC teaching model, which is closer to the traditional online mode of teaching. The behavioral MOOC teaching model is more similar to the existing network teaching model. Most of them are the migration and extension of college teaching models and focus on homework exercises and tests, and provide students with corresponding teaching videos, homework exercises, course exams and other xMOOCs. The teaching mode is shown in figure 2. It is shown that xMOOC teaching mode mainly focuses on the proliferation and replication of knowledge, focusing on the improvement of learning methods such as video teaching, after-school homework, and chapter testing, and is more inclined to the extension of traditional teaching concepts.

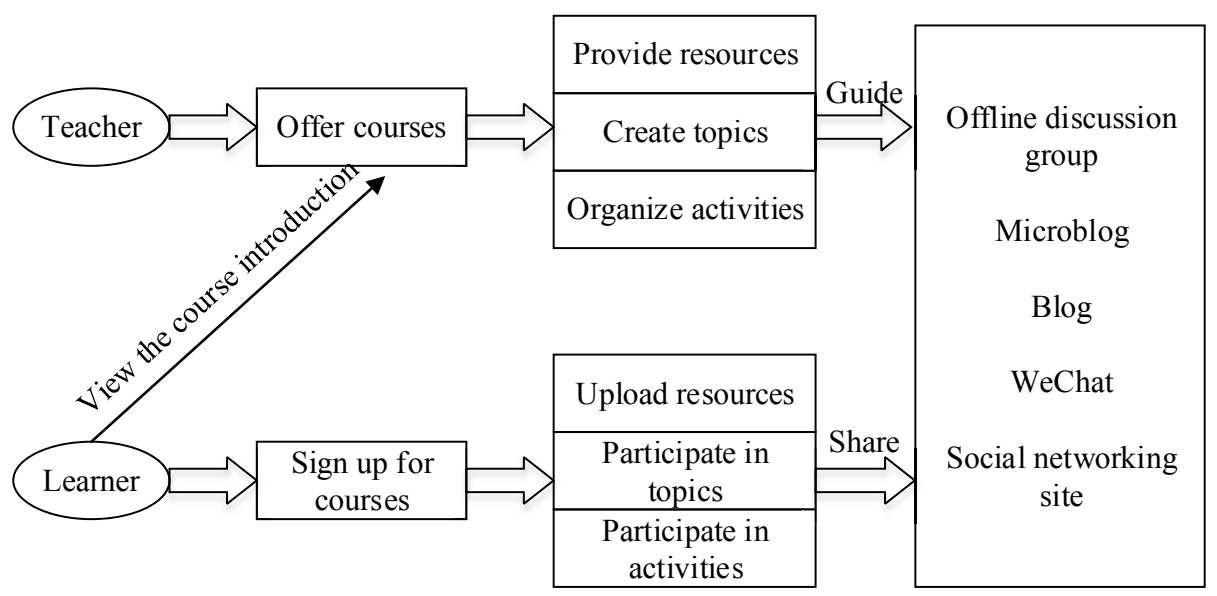

Fig.1. cMOOC teaching mode 


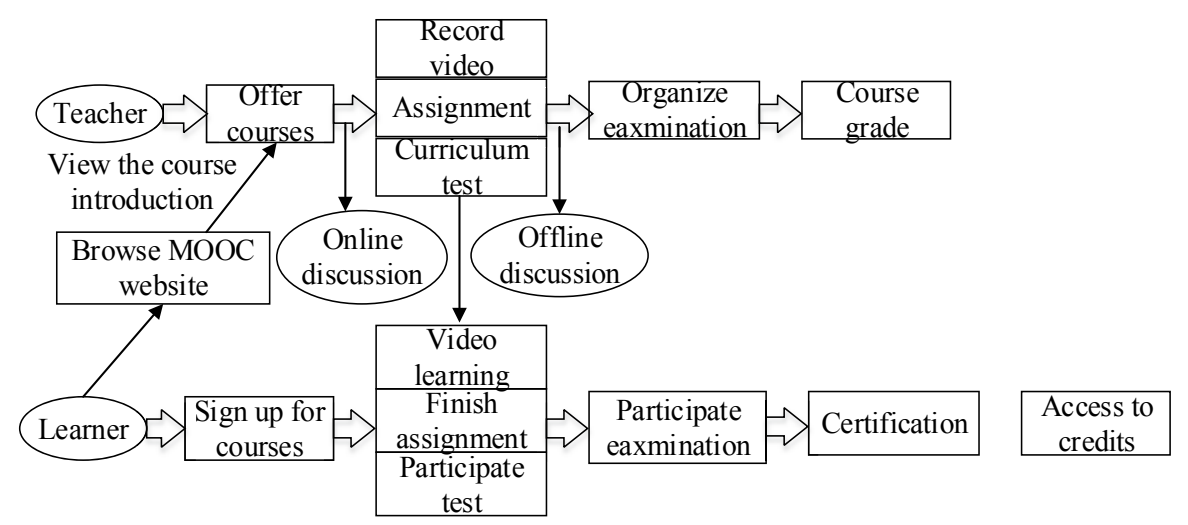

Fig.2. 2 xMOOC teaching mode

\subsection{Feature of MOOC teaching mode}

The difference between MOOC teaching mode and traditional teaching mode is analyzed through the online questionnaire. After analyzing the results of the questionnaire, a bar chart is obtained. As shown in figure 3, the questionnaire results show that the MOOC teaching model not only provides a large number of high-quality, free and online learning courses, but also has a higher requirement for students' autonomous learning ability. This shows that the characteristics of the MOOC teaching model can be summarized as massive, online, open, and course.

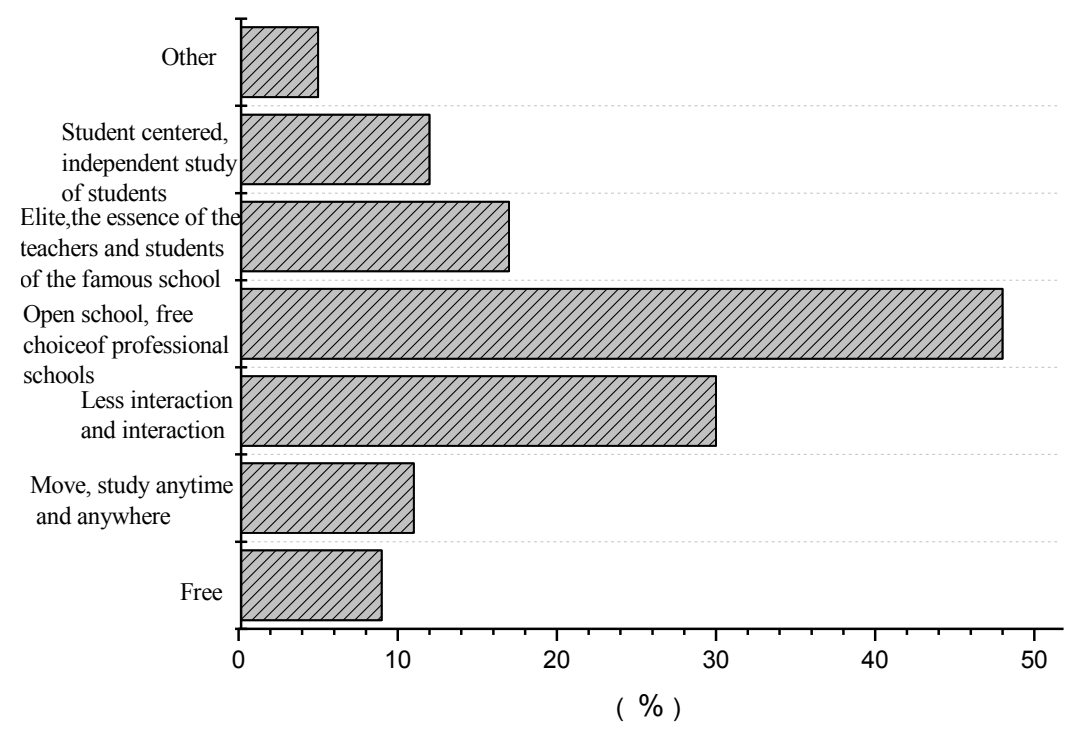

Fig.3. Feature of MOOC teaching mode $\square$ 


\subsection{MOOC virtual exchange center}

On the questionnaire, a corresponding questionnaire survey is conducted on the advantages and disadvantages of the existing MOOC teaching model. The survey results show that although the MOOC teaching model has revolutionary characteristics and great development prospects, it also shows its existing deficiencies. The main shortcomings are single learning environment, poor learning atmosphere, weak interaction, low participation, and single teaching channel. To provide learners with a good learning aid platform, the MOOC virtual exchange center is constructed. It is expected that the lack of existing teaching modes will be compensated by the good immersive, interactive and constructive nature of virtual reality technology. After the learner enters the MOOC virtual exchange center, the system automatically assigns the learner a virtual character as a substitute for the learner. Learners are free to control the behavior of the avatars and move freely in a virtual environment built by virtual reality technology. MOOC virtual exchange center supplements the existing MOOC teaching mode and provides learners with three learning modules: teacherstudent answering module, exploration learning module, and knowledge sharing module. The MOOC virtual exchange center complements the existing MOOC teaching model with respect to learning atmosphere, course participation and learning channels. First of all, from the aspect of learning atmosphere, by simulating the realistic teaching environment and constructing the virtual reality teaching scene, it provides learners with a realistic learning atmosphere. Then, in order to enhance the degree of participation in the course, it provides learners with a real-time learning interaction platform, shortening learners' time to solve problems encountered in the learning process. In terms of enriching learning channels, learners are provided with discussion and communication modes to explore learning modes and knowledge sharing modes so as to achieve better results in accordance with their aptitude. The similarities and differences between the MOOC virtual exchange center and the existing mainstream MOOC teaching platform are shown in table 1. The virtual MOOC exchange center makes up for several deficiencies in the existing MOOC learning model. The main advantages are as follows: The first is to enhance learners' strong immersion. The virtual reality rooms and virtual characters with Unity $3 \mathrm{D}$ are built to create a realistic learning and communication environment. Therefore, learners have an immersive learning experience even through the mouse and keyboard controls. The second is to enhance the real-time interaction between teachers-students, and students -students in the learning process. The virtual MOOC exchange center provides a platform for online learners to explore and learn. At the same time, the utilization of learning resources is enhanced. In the MOOC exchange center, each learner can either learn relevant knowledge as a student or impart knowledge as a teacher to help others learn knowledge. In addition, learners can share their learning experiences and methods with other learners and explore a learning style that suits them in mutual learning. 
Table.1 Comparison table of teaching mode

\begin{tabular}{|l|c|c|}
\hline & $\begin{array}{c}\text { Existing mainstream } \\
\text { MOOC teaching mode }\end{array}$ & $\begin{array}{c}\text { MOOC virtual ex- } \\
\text { change mode }\end{array}$ \\
\hline Large-scale & Yes & Yes \\
\hline Online & Yes & Yes \\
\hline Free & Yes & Yes \\
\hline 3D virtual scene & No & Yes \\
\hline Virtual teacher intelligence answer & No & Yes \\
\hline Virtual exploration learning & No & Yes \\
\hline Virtual multiplayer online communication & No & Yes \\
\hline Virtual real-time knowledge sharing & No & Yes \\
\hline
\end{tabular}

\section{$4 \quad$ Results and discussion}

\subsection{Research of key system technology}

The first is 3D modeling technology. The 3D MAX modeling tool is used. In addition, the real people, classrooms and other scenes are taken as reference to build interactive center simulation environment and character model. In the process of construction, the simplified model facet number is adopted and the light maps are used to render the model. The aim is to reduce the loss of system resources and improve the operating efficiency. After the model is created, the 3D MAX is used to export the model as an FXB file that can be directly imported into the Unity 3D development platform to finish the scene building in Unity 3D later. Unity comes with a powerful graphic rendering function. In order to make the virtual scene more realistic, the Unity $3 \mathrm{D}$ engine is used to deploy the entire MOOC virtual interaction center. From an optimization point of view, Perfabs (models) that is used multiple times in the same scene is created and the instantiate Perfabs is used to build objects.

The second is three-dimensional scene modeling. The design of MOOC virtual exchange center scene model mainly includes the design of tables, chairs, doors, windows, and display boards. In order to improve the utilization of system resources, it is necessary to support the addition of different physical attributes to the model objects imported into Unity. Based on the guarantee of visual effects, this paper uses polygon modeling to minimize the number of model faces and keep the consistence of model. Since the rendering model in 3D MAX will cause distortion when it is imported into Unity, this article will create a good model in 3D MAX and import it into Unity to perform the corresponding rendering. In order to ensure the exquisite texture, the texture map of each material is firstly put into Photoshop for refinement, and the texture file is uniformly saved as the .png format in order to increase the rendering efficiency. The corresponding material is created for each object in Unity and each material is assigned to the object. With adaptive lighting, the desired effect is achieved.

The third is three-dimensional character modeling. In order to enhance the immersive sense of users in the MOOC virtual interaction center, a virtual three-dimensional character is constructed as an embodiment of the teacher and student user in the virtu- 
al scene. The user controls the behavior of the character through the keyboard. At the same time, in order to realistically simulate the action behavior of a character in real life, corresponding character skeletons are added to it and corresponding character animations are made. During the process of model rendering, the rendering method for scene model is used render the corresponding material in Unity. In addition, the character animation is divided to create independent standing, walking, running and jumping animation clips.

The fourth is collision detection technology. For complex irregular models or models with special requirements, the Unity $3 \mathrm{D}$ built-in rule-based colliders are difficult to achieve the desired realistic effect. Therefore, based on the Unity 3D collision detection technology, some improvements have been made. Firstly, the complex irregular model is decomposed into several rule sub-models by the physical model decomposition or the construction of blank sub-item splicing for the model according to its shape features and requirements for collision points. Then, collision detectors are added to each sub-model to achieve a realistic effect that is closer to reality.

\subsection{Development environment and process of MOOC virtual exchange center}

Unity is not only powerful and perfect, it is vivid and lifelike. 3D project development has a short cycle and provides users with a free learning version. It also has a short learning curve and simple interface. The whole operation process is easy to achieve. Whether for beginners or professional development team, it is a good choice. Unity3D MAX has a powerful model construction function, and can produce fine animations, and directly export a variety of formats of models with a strong practicality. Therefore, 3D MAX is selected as the main model building tool in this study. Unity is selected as the main environment for the development of the main system to facilitate the subsequent maintenance and development of the system. The MOOC virtual exchange center development environment is as follows:

Operation system: Microsoft Windows 7

Development environment: Unity 4.5.2

Modeling tool: 3D MAX 2010

Python environment: Python 2.7

Hardware environment: CPU: Intel(R) Core(TM) i5-2410M

In order to build a more realistic and immersive MOOC virtual exchange center, the system uses 3DMAX to model objects and virtual characters in the virtual scene. Then, the Photoshop graphics software is used to process the texture maps needed by the model and save the model as a file format suitable for Unity. The subject is imported into the Unity engine to typeset; its size is adjusted to appropriate $3 \mathrm{D}$ space position. Unity's powerful shader module is used to further render the scene to achieve more realistic effects. After adding corresponding functional components for each object, the physical properties of things in reality are simulated to achieve the expected interactive effects. 


\subsection{Scene design of MOOC virtual exchange center}

The scene design needs to guarantee the certain flexibility and controllability (attracting users to learn for a long time) and clearly demonstrate the basic functions of the system (facilitating users to quickly find the way they want to learn). The MOOC virtual exchange center is roughly divided into three modules: question-answer (Q\&A) module, exploration learning module and knowledge sharing module. The corresponding user flow chart is shown in figure 4.

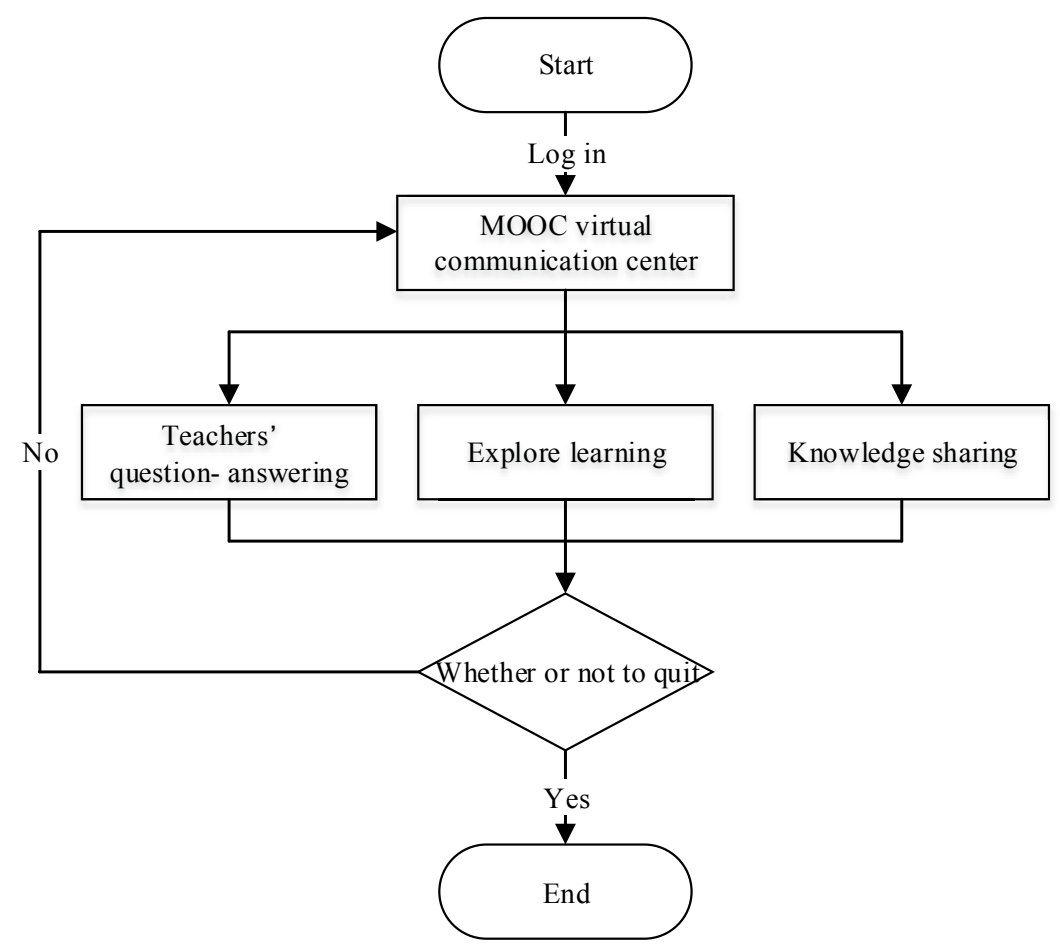

Fig.4. User flowchart of MOOC virtual exchange center

The first is teacher-student Q\&A module scene design. The MOOC virtual exchange center provides learners with a real-time interactive platform for questions and answers. The Q\&A platform is mainly divided into online real-time Q\&A and offline Q\&A. Online real-time Q\&A module refers to constructing virtual question and answer scenes for teachers and students when the teacher is online. Teachers and students communicate in virtual scenes in real time through a face to face approach. The offline intelligent Q\&A module means that when the teacher is offline, the learner obtains the answer from the existing knowledge base in the form of face to face communication with the virtual teacher. Online real-time Q\&A module mainly uses Unity network real-time communication technology to achieve, which is mainly composed of two parts such as problem keyword extraction and keyword-based problem retrieval. 
The second is the scenario design of exploration learning module. The exploration learning module is mainly composed of course information acquisition module, scene roaming module, database reading module and information display module. The course information acquisition module is mainly responsible for obtaining relevant courses provided by the MOOC course information acquisition subsystem, acquiring basic course information and storing it in a database. The scene roaming module is mainly responsible for learners' autonomous exploration activities and automatic exploration activities in the virtual scene. The database reading module is mainly responsible for reading the relevant information of the course from the database. The information display module is mainly responsible for displaying the course information in the virtual classroom.

The third is the design of knowledge sharing module scenarios. Knowledge sharing module structure is divided into knowledge explaining module, knowledge blog module, database module and database access module. The learner can ask questions related to this knowledge lecture in the knowledge lecture, and the creator can give realtime feedback on the question to achieve the effect of knowledge explanation. After entering the lecture hall, learners can search all knowledge blogs related to this topic and learn knowledge in a blog way. In addition, learners can also share their learning experiences or topic-related knowledge to other learners via blogs. Each knowledge point may involve references such as videos, books, documents, and so on. Therefore, the database module mainly provides learners with information related to the topics of the discussion room. Taking into account the operating load of the entire system, the database module will not provide uploading and downloading functions for videos and books, but the web URL of the data for learner. Whether it is a knowledge blog or a database, it requires the support of a database to store data. Therefore, the database reading module mainly stores or reads knowledge blogs and database information from the database.

\subsection{Design and implementation of MOOC course information acquisition subsystem}

The first is the overall design of the MOOC course information acquisition subsystem. The system is implemented by python language, which is mainly divided into three modules: page collection module, page analysis module and data storage module. The system retrieves the URL from the URL queue and uses the page collection module to download the webpage on the MOOC website of the University of China, and then obtains the required content and the related page URL through the parsing of the page analysis module, and then stores the analyzed content in the My SQL database. Finally, the relevant page URL is added to the URL queue. After analyzing the Chinese University MOOC website, the demand for the existing MOOC course information is explored based on the MOOC virtual interaction center.

The second is the page acquisition module. The types of web pages that need to be collected are divided into two types according to the user's access rights: regular web pages and personal web pages. A regular web page means that users can view web page content as a visitor. A personal web page means that a user must log in to view 
web content before browsing. The function of collecting page information in this article is mainly achieved through python's urllib library. The main task of the page analysis module is to analyze the webpage data collected by the webpage, filter the content through a specific regular expression, and obtain the data and URL that users want. In the process of content analysis, feature extraction is first performed on the content of the web page to be acquired by the system, and a specific regular expression is formulated according to the feature. Then, regular expression is used to extract the data. If the content extraction is failed, the regular expression needs to be corrected and re-enactment.

The third is the data storage module. The effective data after analysis is stored and used for other modules. It is mainly divided into two parts: storing valid content data into a My SQL database for use in a MOOC virtual classroom and adding a valid URL to a URL queue for use by a page collection module.

\subsection{Operation effect of MOOC virtual exchange center}

MOOC virtual exchange center provides users with a self-learning threedimensional virtual platform. As a supplement of the existing MOOC teaching platform, the MOOC virtual exchange center mainly provides learners with three scenarios such as answering questions, exploring learning and sharing knowledge. Teachers and students answer scenes are mainly divided into online real-time Q\&A and offline Q\&A. Online real-time answering refers to constructing virtual question and answer scenes for teachers and students when teachers or learners with the same direction are online. Teachers and students communicate in virtual scenes in real time through a face to face approach. The offline intelligent question answering means that when the teacher is offline, the learner obtains the answer from the existing knowledge base in the form of face-to-face communication with the virtual teacher in the virtual classroom. It includes independent exploration learning and automatic exploration learning. In the autonomous exploration learning mode, the learner enters the virtual scene and simulates the state in which the learner enters the teaching building. The learner can freely enter the virtual classroom of the existing course, preview the corresponding course knowledge, and explore the courses they are interested in. In the automatic exploration learning mode, learners can select existing popular MOOC courses after entering the scene and automatically roam to the corresponding classroom for learning. After entering the MOOC virtual exchange center, learners can choose the knowledge sharing module to create corresponding knowledge lectures based on their own needs, and to perform corresponding knowledge explanations as teachers, or to share static knowledge by writing knowledge blogs and uploading learning materials.

\section{Conclusion}

The MOOC teaching model based on virtual reality can combine the advantages of face-to-face classroom teaching and online learning to achieve the optimal teaching effect. Through the introduction of MOOC teaching mode, the characteristics and 
disadvantages of this education model are analyzed in depth. A combination of MOOC education model and virtual reality technology is proposed to construct a teaching platform based on MOOC virtual exchange center. The advantages of the MOOC teaching model based on virtual reality are analyzed. In short, with the continuous development of networked education, higher requirements are placed on the learning platform, and the MOOC teaching model based on virtual reality technology is a new attempt, opening up a new idea for the future of online education research.

\section{Acknowledgement}

1 Dali University 2016 doctoral research project: the value, inspiration and application of embodied cognition to curriculum and teaching (BSKY201605)

2 Dali University Sixth phase of education and teaching reform project "Research on Teacher Education Curriculum Construction Based on core literacy"

\section{$7 \quad$ References}

[1] Borras-Gene. O., Martinez-Nuñez, M., \& Ángel Fidalgo Blanco. (2016). New challenges for the motivation and learning in engineering education using gamification in mooc. International Journal of Engineering Education, 32(1(B)): 501-512.

[2] Zeming, L. (2017). Design and implementation of a korean language teaching system based on virtual reality technology. Agro Food Industry Hi Tech, 28(1): 2156-2159.

[3] Dehua, W., Dehua, W., \& Dehua, W. (2017). The teaching resource development analysis of micro learning resource and mooc in information teaching. Revista De La Facultad De Ingenieria, 32(3): 271-278.

[4] Feng, T. (2017). Research on teaching model of mooc-based college english flipped classroom. Boletin Tecnico/technical Bulletin, 55(20): 503-508.

[5] Jia, H., \& Ma, D. (2014). Problems concerning MOOC's development in China. Journal of Hubei Engineering University, 34(4):76-80.

[6] Li, C., \& Zhou, H. (2018). Enhancing the efficiency of massive online learning by integrating intelligent analysis into moocs with an application to education of sustainability. Sustainability, 10(2): 468. https://doi.org/10.3390/su10020468

[7] Cao, F. (2016). A ship driving teaching system based on multi-level virtual reality technology. International Journal of Emerging Technologies in Learning, 11(11): 26. https://doi.org/10.3991/ijet.v11i11.6249

[8] Li, H., \& Li, H. (2017). Design of multimedia teaching platform for chinese folk art performance based on virtual reality technology. International Journal of Emerging Technologies in Learning, 12(9): 28. https://doi.org/10.3991/ijet.v12i09.7487

\section{Authors}

Yongfei Zhang is with College of Education Science, Dali University, Dali, Yunnan, China.

Jianshu Chen is with the Department of Public class, Honghe Health Vocational College, Mengzi, Yunnan, China. 
Dan Miao is with the Department of Public class, Honghe Health Vocational College, Mengzi, Yunnan, China.

Chen Zhang is with the Department of Pharmacy, Honghe Health Vocational College, Mengzi, Yunnan, China.

Article submitted 26 April 2018. Final acceptance 07 May 2018. Final version published as submitted by the authors. 